\title{
Effective Geographical Routing in the Presence of Unpredictable Node Mobility
}

\author{
P. Srimanchari \\ Assistant Professor \\ Department of Computer \\ Science Erode Arts \& Science \\ College
}

\author{
M. Saroja,Ph.D \\ Associate Professor \\ Department of Electronics \\ Erode Arts \& Science College
}

\author{
M. Venkatachalam,Ph.D \\ Professor \& HOD \\ Department of Electronics \\ Erode Arts \& Science College
}

\begin{abstract}
The geographical routing protocol employs position information from the localization system for multi-hop data communication. However, the location inaccuracy due to the unpredictable node mobility and the power consumption of localization system reduces the performance of geographical routing protocol. Several geographical routing protocols have been proposed in Mobile Ad Hoc Network (MANET). The well-known geographical routing among them is Greedy Perimeter Stateless Routing (GPSR). In GPSR, the dead end node selection for data communication easily handles the network scalability. However, the highly dynamic network topology makes frequent changes in the neighbor's location. Hence, the inaccurate location information degrades GPSR performance. This paper proposed a Modified GPSR (MGPSR) protocol to improve the performance under a large scale and a high mobility network. Moreover, the MGPSR consists of Neighbor List Learning (NNL), Node Mobility Prediction (NMP), and Periodic Position Update (PPU) schemes to balance the data delay and packet delivery ratio. The NNL along with NMP scheme provides the accurate neighbor list under a high mobility network. Furthermore, the PPU supports the guarantee for accurate neighbor location. In addition, the proposed schemes reduce the usage of localization systems to provide accurate location information with low power consumption which improve the routing performance in MGPSR. This work simulates and compares the performance of an enhanced MGPSR in the aspects of packet delivery ratio and throughput with the existing GPSR. Thus, it proves the MGPSR outperforms the GPSR under a large scale with a high mobility network.
\end{abstract}

\section{Keywords}

Mobile Ad Hoc Network, Geographic Routing, Location Accuracy, and Localization System, GPSR

\section{INTRODUCTION}

Geographical routing protocols present a different approach from the existing topology based routing. It eliminates the reliability of topology storage since; it uses the physical location information to take the routing decision. In geographical routing protocols, no need to maintain end-to-end route information. Hence, it can easily handle the dynamic behavior of network topology and scale extremely well for a large network [1]. The well-known geographical routing protocol in MANET is GPSR [2]. The GPSR includes two kinds of data forwarding such as Greedy and Perimeter routing. Initially, it activates the greedy mode which makes the location based localized routing decision to transmit data packets. Moreover, to obtain the node location it relies on GPS or any other localized location services [3]. In addition, it periodically broadcast the beacon messages within communication range to retain the location of radio neighbors. Furthermore, it selects a neighbor node, which is located close to the destination node to transmit data packets.
The inaccurate location service and location inaccuracy due to the unpredictable node mobility degrades the performance of geographical routing protocols [4]. Moreover, the GPS has poor accessibility on the mobile node, and it utilizes high power, which affects the GPSR performance. In addition, the neighbor list retained at the mobile node is outdated when a new node enters or leaves from the node communication range. As a result, the data packets are lost when uses the outdated location information to forward the data packets. To avoid the packet loss due to the outdated location information, the node neighbor list needs to update periodically. Hence, the GPSR relies beacon messages to ensure the neighbor list frequently. Moreover, the periodic broadcast of beacon messages during the data communication incurs beacon overhead and collision. However, the network scalability increases the number of beacon messages, and the node mobility increases the location inaccuracy. Hence, this leads to the poor performance of GPSR [5].

To improve the GPSR performance under a highly dynamic network topology, a modified GPSR along with the NNL, NMP, and PPU schemes is proposed for MANET. The NNL learns the accurate neighbor list and NMP predict the node mobility to reduce the packet loss. Moreover, the PPU uses controlled beacon messages to provide guarantee to the location accuracy. It aids to mitigate the GPS or any other location service usage. Hence, the proposed protocol MGPSR along with the NNL, NMP, and PPU enhances the GPSR performance. Moreover, this work compares and simulates the performance of MGPSR in terms of packet delivery ratio and throughput to prove that the MGPSR outperforms the GPSR.

\subsection{Problem Statement}

The most of the prominent geographic routing protocols in MANET takes a routing decision based on the location information. GPSR is a geographic routing protocol; it selects the dead end nodes based on the greedy routing to forward the data packets. As a result, the GPSR optimal node selection for a data transmission makes it suitable to the large scale networks. However, the location inaccuracy due to the highly unpredictable node mobility degrades the GPSR performance. Moreover, the GPSR uses the GPS or other localized services to identify the node location. However, the GPS has several disadvantages under MANET for instance; it consumes high power and may not be accessible on all the mobile nodes. Hence, the GPS or any other localized services make a negative impact on the greedy routing. Therefore, to improve the GPSR performance under a highly dynamic network topology, GPS or other location service usage should be reduced to recognize the location error possibility. Hence, to improve the routing performance the MGPSR is proposed along with the NNL, NMP, and PPU schemes for MANET. According to the dynamic network the MGPSR obtains and updates the local topology information using GPS and beacon messages. In addition, the proposed schemes use beacon messages to the neighbor list identification and the mobility prediction which aids to provide a 
guarantee for location accuracy. As a result, it successfully balances GPSR [13], Power Efficient GPSR [14], and an Energy Aware the power utilization and the transmission delay. Thus, the MGPSR Greedy Routing Scheme (EAGR) [15] are proposed to consume the performs well under a large scale network with highly dynamic node energy. mobility.

\subsection{Contributions}

The contributions of the paper are:

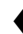

This work studies the performance geographical routing in the presence of inaccurate location service due to the node mobility.

The proposed work extends GPSR along with the NNL, NMP, and PPU mechanisms to enhance the performance. The accurate neighbor location information for greedy routing is obtained using the NNL under a highly dynamic network to enhance the routing performance. The unwanted bacon broadcast is avoided using NMP scheme. The periodic broadcast of beacon messages in PPU controls the beacon overhead and the beacon collision. The balance among the communication delay and the power utilization are achieved using the various beacon intervals corresponding to the node state and the neighbor priority.

The performance of the proposed MGPSR is simulated and compared with the GPSR in terms of packet delivery ratio and throughput.

\subsection{Paper Organization}

The paper is arranged as follows: The chapter 2 provides the idea of the previous work which is related to the GPSR. The chapter 3 proposes the Modified GPSR along with the PPU routing scheme under MANET. The chapter 4 demonstrates the experimental results for MGPSR performance. The chapter 5 concludes the work.

\section{RELATED WORKS}

This part illustrates the existing works on geographical routing protocol such as GPSR. It describes the effect of location services and the location inaccuracy due to the node unpredictable mobility on GPSR performance.

\subsection{Existing Geographical Routing Protocols}

Geographical routing protocols and its drawbacks are surveyed in [6]. The geographical routing does not need topology storage, which makes it suitable to the dynamic network topologies. However, mobility, location accuracy, power consumption, and the QoS requirement affect the existing geographical routing protocols. A scalable location based routing method [7] and the compass routing [8] [9] are aids to improve the greedy routing. In addition, the traversal steps to the perimeter path detection are presented in [10]. However, it incurs additional overhead to the routing. The routing protocol for the reliable data delivery under a large scale with a high mobile network is presented in [11]. It provides the position based opportunistic routing, which utilizes the advantages of geographical routing stateless property. It reduces the duplicate relaying and data delay due to the location inaccuracy.

To reduce the location inaccuracy the Small World Iterative Navigation Greedy Routing (SWING) protocol is proposed in [12]. Even in the network with inaccurate location information, it uses the greedy routing to the successful data delivery. Moreover, it recovers the sub optimality problem due to the void routing. However, it consumes high power to provide the efficient data delivery. Since, the selection of a node for frequent data transmission leads to energy loss. Hence, the Energy Aware E-

\subsection{Location Service and its Effect on Geographical Routing}

The location based geographical routing needs external location services to obtain the node physical location. A scalable location service for geographical routing such as Grid Location Service (GLS) is presented in [16]. It constructs the network as scalable to provide the efficient geographic routing under a large scale network. To provide the Network Environment Wireless State (NEWS) service to MANET geographical routing, the Legend Exchange and Augmentation Protocol (LEAP) are proposed in [17]. Moreover, it maintains the local and global data bases to provide accurate location information without increase the routing overhead. However, it consumes high power and also it is not accessible on all the scenarios.

\subsection{Mobility Effect on Geographical Routing}

The mobility induces the location inaccuracy which leads to insufficient geographical routing performance [18]. It provides Neighbor Location Prediction (NLP) and Destination Location Prediction schemes to mobility induced problems such as loop and link failure. In [19], the impact of mobility induced location errors on geographical routing is illustrated. Moreover, an effect of localization errors on geographical routing is presented in [20]. Furthermore, to enhance the geographical routing protocols, the effect of node mobility is considered in [21]. In that, the concept of motion potential is incorporated in node mobility patterns to make an effective routing decision. Hence, these solutions predict the node mobility to reduce the effect of the node mobility effect on geographical routing.

In order to reduce the effect of external location service, and mobility induced location inaccuracy, the GPSR need to incorporate an efficient routing technique into the routing. Since, the high power consumption of external location services and the location inaccuracy effect on greedy routing degrades the GPSR performance. Moreover, several solutions are provided to these issues. Hence, new routing schemes provide solutions to the issues to balance the power consumption and data delays.

\section{MODIFIED GPSR}

A new MGPSR is proposed to improve the GPSR performance under a large scale network with highly dynamic topology. In MGPSR, assume all the mobile nodes know their own location information using GPS or any other location services. Moreover, the MGPSR contains two data forwarding modes such as Greedy and Perimeter like GPSR. In MGPSR, every node broadcast the beacon messages to their radio neighbors to obtain the neighbor list. If a sender node has radio neighbors, it activates the greedy mode otherwise, activates the perimeter mode to forward data packets. The greedy data forwarding is shown in Fig 1. In that, the sender node $\mathrm{S}$ selects a node which is nearer to the destination to forward the data packets. As a result, the data packets are delivered to the destination successfully under a large scale network 


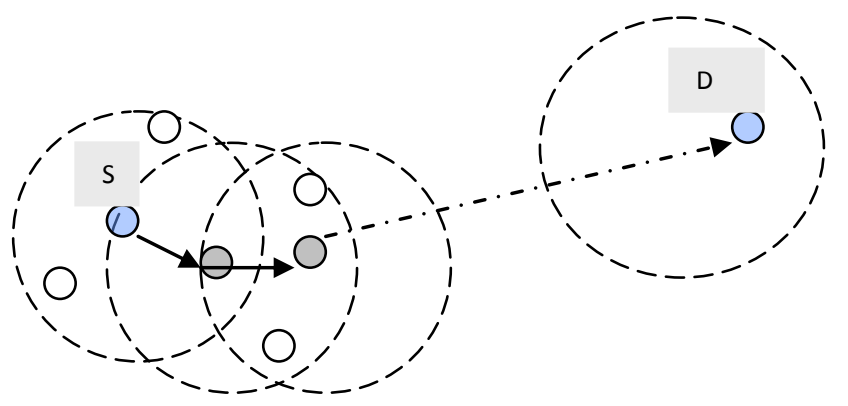

Fig 1 : Greedy Forwarding

The data packets are lost where a node cannot determine any neighbor to forward the packets called Local Maximum. Hence, the GPSR activates the perimeter mode to forward the data packets. Furthermore, the perimeter mode is changed to the greedy forwarding mode when a node has radio neighbors towards the destination. However, the neighbor list may retain inaccurate location information due to the unpredictable node mobility resulting in poor performance. To improve the performance under a highly dynamic network topology, the MGPSR proposes scheme such as the NNL, NMP, and PPU to retain the accurate neighbor list. Moreover, it aids to balance the power utilization and the communication delay.

\subsection{Neighbor List Learning}

Upon a preliminary state, every node obtains the physical location information using GPS or any other location services. Moreover, the geographical routing requires the neighbor node's information to make the routing decision. Hence, each node broadcasts one hop-beacon messages to obtain the neighbor's location information within the communication range. It stores the local topology information or received beacon information from the neighbors is represented as a neighbor list. In geographical routing, each node provides the data forwarding possibility only to the nodes in the neighbor list [22]. Every node broadcast one hop-beacon message to identify the neighbor's distance towards the destination is shown in the equation (1). In that, the node forwards constant velocity $(\gamma)$ beacon messages per constant interval $(\mu)$ to minimize the energy consumption. In addition, the beacon message estimates and enters the distance from the neighbor nodes to the destination into the neighbor list. Moreover, the neighbor nodes are entered into the list based on the traveling time of the beacon $\left(\tau_{\mathrm{BR}}\right)$ shown in the equation (2). However, the neighbor list obtains frequent changes due to the node mobility. As a result, a high mobility network incurs packet loss. Hence, the NMP is proposed to predict the node mobility which reduces the packet loss.

$$
\begin{aligned}
& \mathrm{NH}_{\mathrm{I}}=\left\{\mathrm{D}_{(\mathrm{NHI}-\mathrm{D})}\right\} \ldots \ldots \ldots(1) \\
& \tau_{\mathrm{BR}}=\mathrm{D}_{(\mathrm{NHI}-\mathrm{D})} /(\gamma \times 1000) \mathrm{ms} \ldots \ldots(2) \\
& \mu=2 \tau_{\mathrm{BR}} /(\gamma \times 1000) \mathrm{ms} \ldots \ldots \ldots(3)
\end{aligned}
$$

\subsection{Node_Mobility Prediction Scheme}

Each node predicts its mobility to reduce the location list frequently. It is because; the nodes physical location is changed inaccuracy routing [23] using NMP scheme. Moreover, it tracks the frequently. Hence, the PPU is proposed to ensure the location neighbor's movement and prospective quadrant. Hence, it aids to accuracy to forward the data packets. To improve GPSR reduce the packet loss and the number of unwanted beacon performance, the PPU provides adaptability to the high mobility transmission to the neighbor node which is going to move. In NMP, network. In that, the beacons play a significant role to provide every node includes the column for the neighbor's mobility characteristic in the beacon header is called NMP flag. In NMP, every node divides the network into four quadrants to predict the node mobility direction. If the predicted position of a neighbor node is within the destination quadrant and it obtains the positive progress movement, the node set the NMP flag as negative. Moreover, the flag is set as positive when it predicts the negative progress mobility from the destination. Furthermore, the neighbor node is considered as a temporary neighbor $\left(\mathrm{T}_{\mathrm{NH}}\right)$ when the NMP flag is positive. If it is positive, the sender node is ready to change the priority or to delete the $\mathrm{T}_{\mathrm{NH}}$ information from the list. Furthermore, the sender node does not select $\mathrm{T}_{\mathrm{NH}}$ node to forward the beacon messages and data packets since; the node will obtain the negative progress movement. Thus, the NMP scheme balances the communication delay and the power consumption.

Fig 2 : NMP Scheme

$\mathrm{PX}_{\mathrm{I}}=\mathrm{X}_{\mathrm{I}}+\left(\tau_{\mathrm{BR}} \times \mathrm{B}_{\mathrm{VI}}\right) \ldots \ldots \ldots$

Consider the node I which divides the network into four quadrants. In that, the destination quadrant is considered as a first quadrant. Then, the node predicts the $\mathrm{X}$ and $\mathrm{Y}$ axis using the equation (4) and (5). In that, the predicted location is within the first quadrant, and it moves toward the destination. Hence, the node set the NMP flag is negative. Moreover, the sender node does not delete the node_I details from the list. However, the NLL along with the NMP scheme provides an accurate neighbor list for greedy routing, not an accurate location information. Since, the highly dynamic nature of mobile nodes affects the location accuracy. To mitigate the mobility effect on location accuracy, the PPU scheme is proposed.

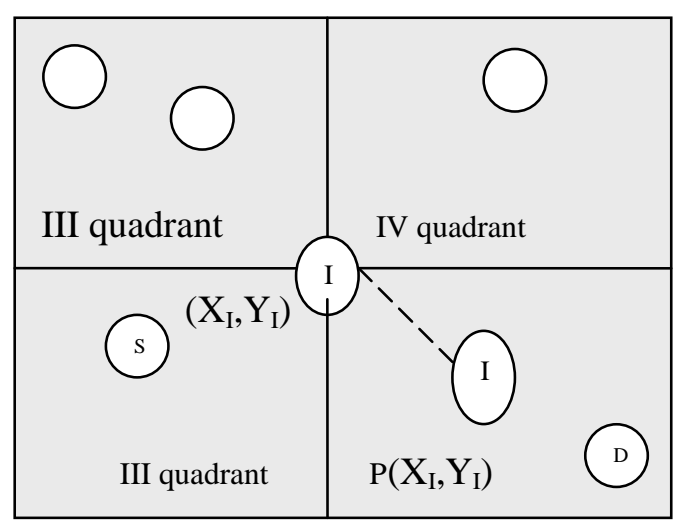


guarantee to the location accuracy without using any location services. Moreover, the nodes during the data communication need to send high velocity beacons frequently to reduce the communication delay. In active nodes the small beacon interval leads to the location accuracy but, consumes more energy. In idle nodes, the large beacon interval for neighbor nodes effect location accuracy. However, low velocity beacons are sufficient to ensure the accuracy of neighbor node's position which aids to reduce the power consumption. Hence, the PPU scheme considers the node conditions individually to balance the communication delay and energy consumption. The PPU under idle mode is called idle mode PPU and active mode PPU under active mode.

\subsubsection{Idle_Periodic Position Update (I_PPU)}

If a node is in idle state, it uses beacon messages to ensure the accuracy of neighbor list. Moreover, forwards the beacon messages to verify the neighbor's location as convenient as the NLL. The neighbor nodes append the position information in the beacon message. Then, the neighbor node send back the beacon message to the sender node using the same velocity. Then, the sender node compares the received beacon information with its neighbor list. If any changes in the received information, it updates the neighbor list.

\subsubsection{Active_Periodic Position Update (A_PPU)}

In the active state, need for fast location accuracy is essential when the sender node demands communication with the destination. Sender node broadcast various high velocity beacon messages to the neighbor nodes to vary the receiving time of beacon reply of radio neighbors. It aids to balance the communication delay and energy consumption. Moreover, the traveling time of the beacon message is determined using the equation (5). However, the beacon velocity $\left(B_{V}\right)$ is determined using the equation (6). For instance, the first priority node velocity equal to the ratio of distance to the destination to the $\tau_{\mathrm{D}}$ which is calculated using the equation (4). As a result, the sender node receives the higher priority node reply within a Milli Second and receives the next priority node reply within 1.5 Milli Second. Hence, the node velocity increases corresponding to the node routing priority. After receiving the first reply, node forward data packets towards the destination. Thus, the MGPSR greedy routing improves the routing performance under a highly dynamic network topology.

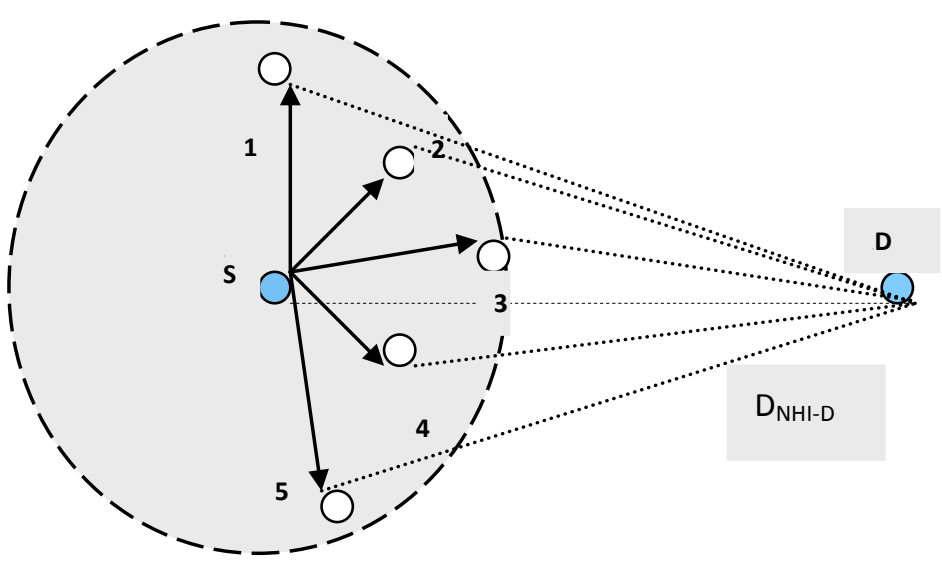

Fig 3 : PPU Scheme

$$
\begin{aligned}
& \tau_{\mathrm{D}}=\mathrm{D}_{(\mathrm{S}-\mathrm{NHI})} /\left\{\mathrm{NH}_{\mathrm{I}}\right\} \mathrm{ms} \ldots(4) \\
& \tau_{\mathrm{BR}}=\mathrm{D}_{(\mathrm{S}-\mathrm{NHI})} /\left(\tau_{\mathrm{D}}\right) \mathrm{ms} \ldots \ldots(5) \\
& \mathrm{B}_{\mathrm{V}}=\mathrm{D}_{(\mathrm{S}-\mathrm{NHI})} / \quad \tau_{\mathrm{BR}} \ldots \ldots(6) \\
& \mathrm{D}_{(\mathrm{NHI}-\mathrm{D})}=\mathrm{D}_{(\mathrm{S}-\mathrm{D})}-\mathrm{D}_{(\mathrm{S}-\mathrm{NHI})} \ldots(7)
\end{aligned}
$$

In Fig 3, the PPU scheme is demonstrated. In that, the sender node $\mathrm{S}$ broadcasts the $\gamma$ velocity beacons to the neighbors to obtain the location information when it is in idle state. After that, the node $\mathrm{S}$ estimates the distance to the neighbor nodes and the destination using the equation (7). Then, a node $S$ sends various velocity beacon messages to the neighboring nodes. For example, the node _3 velocity is higher than the node _2. Hence, the node_3 reply is reached before the node_2. It aids to mitigate the effect of node mobility on routing. For example, the sender node does not obtain a reply from the node_3 when it is moved. The sender node waits until it receives the node_2 beacon reply. On the receipt of the beacon reply, the greedy data forwarding is activated in node_2. As a result, it avoids the packet loss and reduces the communication delay. Thus, it improves the GPSR performance under a large scale with a high mobility network. 


\section{PERFORMANCE EVALUATION \\ 4.1 Simulation Model}

The proposed MGPSR protocol is analyzed and compared with the GPSR using the NS2 simulator.

\subsection{Simulation Parameters}

The simulation model scenario consists of 100 nodes connected using TCP to evaluate the protocol performance. The simulation parameters are listed as under:

Table 5.1: Simulation Parameter for Performance Evaluation

\begin{tabular}{|l|l|}
\hline Software for simulation & N/W Simulator 2 \\
\hline Channel & Wireless \\
\hline Simulation runs time & 200 seconds \\
\hline Area & $500 X 500$ \\
\hline Packet size & 1024 bytes \\
\hline Transport Agent & TCP \\
\hline Pause Time & $0-100$ sec \\
\hline Speed & $0-20 \mathrm{~m} / \mathrm{s}$ \\
\hline Antenna Type & Omni Antenna \\
\hline
\end{tabular}

\subsection{Simulation Results}

The performance evaluation of the MGPSR protocol is simulated to prove its performance. The performance is evaluated in terms of packet delivery ratio and throughput.

\section{Packet Delivery Ratio}

The packet delivery ratio of the GPSR is reduced due to the location inaccuracy. The highly dynamic network topology changes the neighbor list frequently. Moreover, the GPSR uses the external location services to obtain the nodes physical location. Thus, it consumes high energy. As a result, the packet delivery ratio is decreased.

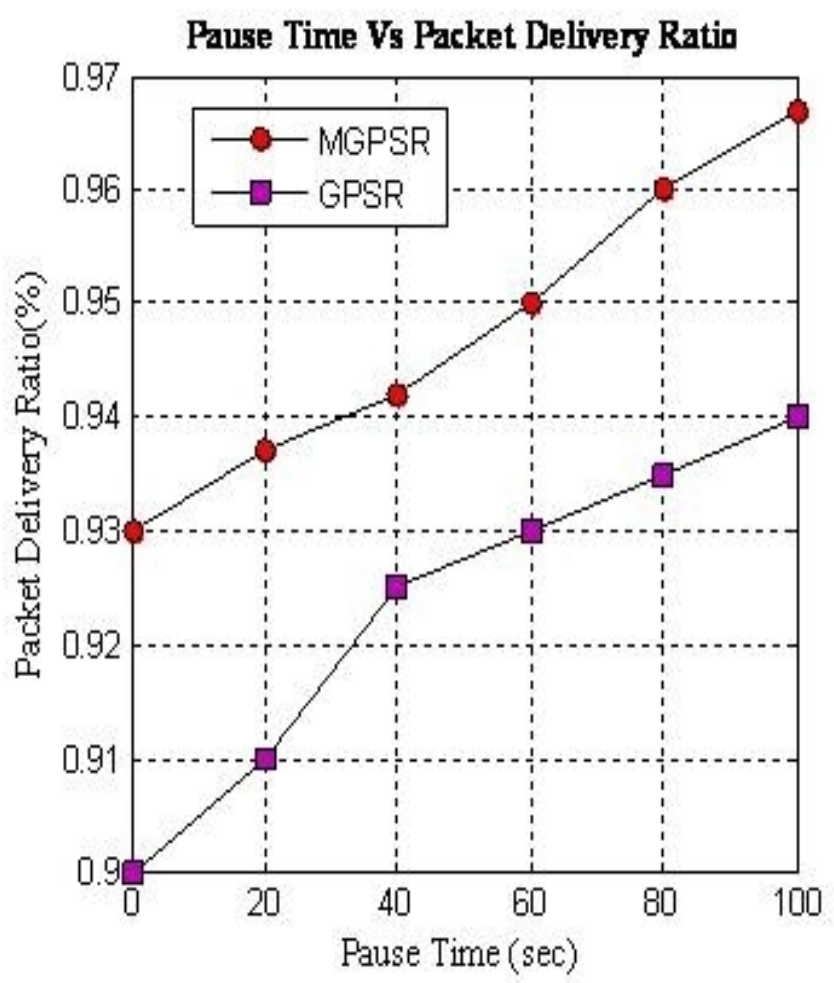

Fig 4. Pause Time Vs Packet Delivery Ratio

In MGPSR, the proposed scheme NLL, NMP, and PPU balances the power consumption and the data delay. Hence, it avoids the packet loss resulting in the efficient packet delivery ratio shown in Fig 4. The packet delivery ratio of MGPSR is $93 \%$ for 0 second pause time and $96.7 \%$ for 100 seconds pause time.

\section{Throughput}

The throughput is defined as the amount of transmitted data packets to the destination node over a period represented in kilobits per second (kbps). In GPSR, the data delay due to the high node mobility induces the location inaccuracy is increased. Moreover, owing to the unwanted transmission of beacon messages and data packets induces the data delay which increases the number of waiting packets. As a result, the throughput rate is reduced. To reduce the unwanted packet transmission, MGPSR predicts the node mobility which aids to reduce the data delay. Hence, it maintains the throughput rate successfully shown in Fig 5. The throughput of MGPSR is $8.8 \mathrm{kbps}$ for 0 second pause time, and $9.28 \mathrm{kbps}$ for 100 seconds pause time. 


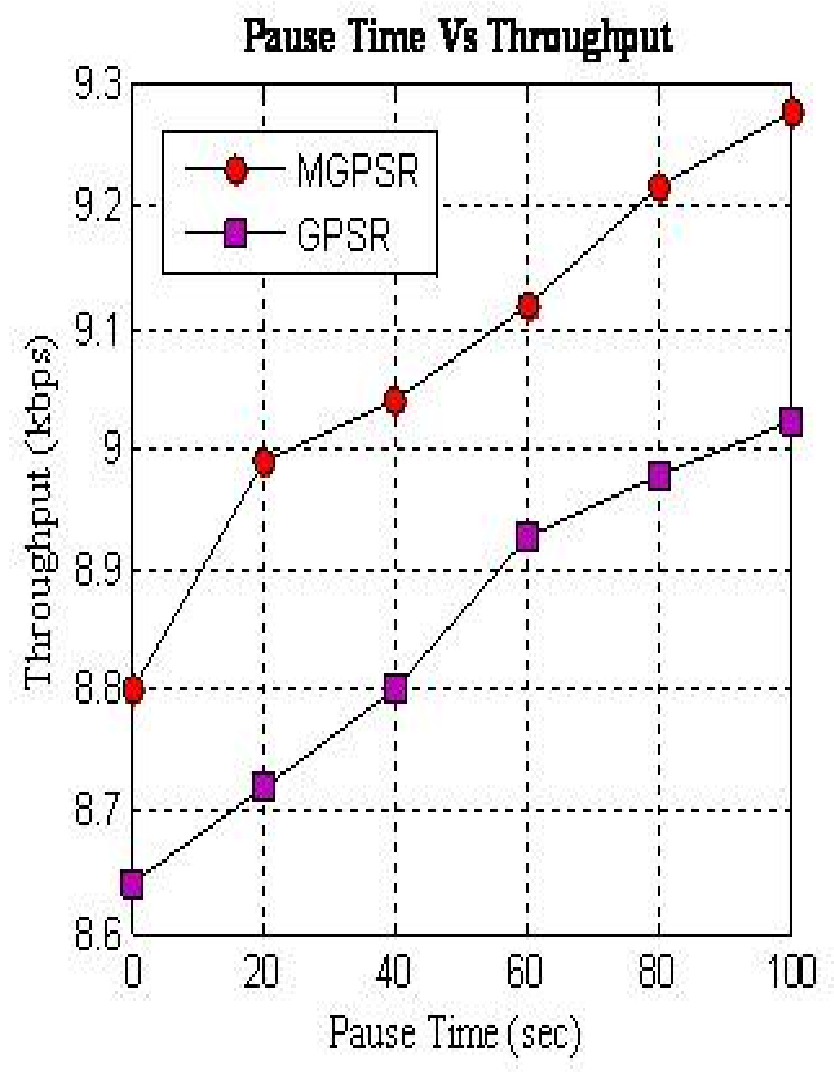

Fig 5. Pause Time Vs Throughput

\section{CONCLUSION}

This paper presented the performance issues of GPSR protocol such as location inaccuracy and external location services under a large scale with a high mobility network. Moreover, it proposes MGPSR protocol which includes routing schemes such as NLL, NMP, and PPU to enhance the GPSR performance. Furthermore, the NLL is utilized to learn the neighbor list for greedy data forwarding. In addition, the NMP is used to predict the node mobility to reduce the packet loss. Moreover, the PPU is used to balance the data delay and the power consumption. Hence, the MGPSR performance is enhanced under a large scale with a high mobility network. Moreover, the performance of the proposed MGPSR protocol is simulated and compared with the GPSR. Thus, the simulation results prove that the MGPSR outperforms the GPSR.

\section{REFERENCES}

[1] Young-Jin Kim, Ramesh Govindan, Brad Karp, Scott Shenker, "Geographic routing made practical", Proceedings of the 2nd Conference on Symposium on Networked Systems Design \& Implementation, Vol 2, pp 217-230, 2005.

[2] B. Karp and H.T. Kung, "GPSR: Greedy Perimeter Stateless Routing for Wireless Networks", Proceedings of the Sixth Annual ACM/IEEE International Conference on Mobile Computing and Networking, pp 243-254, 2000.

[3] Julio C. Navas and Tomasz Imielinski, "GeoCast - Geographic Addressing and Routing", Proceedings of the 3rd Annual ACM/IEEE International Conference on Mobile Computing and Networking, pp 66 - 76, 1997.

[4] Kaream Seada, and Ramesh Govindan, "On the Effect of Location Inaccuracy on Geographic Face Routing in Wireless Networks", IEEE Transactions on Ad Hoc Networks, Vol. 5, No. 6, pp. 855-871, 2007.

[5] Raed Alsaqour and Maha Abdelhaq, "Analysis of Mobility Parameters Effect on Position Information Inaccuracy of GPSR Position-Based Manet Routing Protocol”, Vol 28, No.2, pp 114-120, 2011

[6] Fraser Cadger, Kevin Curran, and Sandra Moffett, "A Survey of Geographical Routing in Wireless Ad-Hoc Networks", IEEE Communications, Vol. 15, No. 2, pp 621 - 653, 2013.

[7] Ljubica Blazevic, Jean-Yves Le Boudec, and Silvia Giordano, "A Location-Based Routing Method for Mobile Ad Hoc Networks", IEEE Transactions on Mobile Computing, Vol. 4, No. 2, pp 97-110, 2005.

[8] Evangelos Kranakis, Harvinder Singh, and Jorge Urrutia, "Compass Routing on Geometric Networks", Proc Canadian Conference on Computational Geometry, pp 51-54, 1999.

[9] Hannes Frey and Ivan Stojmenovic, "On Delivery Guarantees of Face and Combined Greedy-Face Routing in Ad Hoc and Sensor Networks", Proceedings of the 12th Annual International Conference on Mobile Computing and Networking, pp 390-401, 2006.

[10] Chia-Hung Lin, and Ming-Jer Tsai, "ProgressFace: An Algorithm to Improve Routing Efficiency of GPSR", IEEE Transactions on Computers, Vol.59, No. 6, pp 822-834, 2010.

[11] Shengbo Yang, and Bu Sung Lee, "Toward Reliable Data Delivery for Highly Dynamic MANET", IEEE Transactions on Mobile Computing, Vol. 11, No. 1, pp 111-124, 2012.

[12] A.S. Ahn, A.T. Campbell, A. Veres and L. Sun, "Supporting Service Differentiation for Real-Time and Best Effort Traffic in Stateless Wireless Ad Hoc Networks", IEEE Transactions on Mobile Computing, Vol.1, No.3, pp. 197-207, 2002.

[13] Natarajan Meghanathan, "An Energy-aware Greedy Perimeter Stateless Routing Protocol for Mobile Ad hoc Networks", International Journal of Computer Applications, Vol. 9, No.6, pp 30-35, 2010. 
[14] Lan Luan, Wen-Jing, Hsu and Rui Zhang, "Power-Efficient Geographic Routing for MANETs", Journal of Information Science and Engineering, Vol 20, No 1, pp.157-180, 2004.

[15] Sachin Sharma, H.M. Gupta, and S. Dharmaraja, "EAGR: Energy Aware Greedy Routing Scheme for Wireless Ad hoc Networks", International Symposium on Performance Evaluation of Computer and Telecomm Systems, pp. 122-129, 2008.

[16] Jinyang Li, John Jannotti, Douglas S. J. De Couto, David R. Karger, and Robert Morris, "A Scalable Location Service for Geographic Ad Hoc Routing", Proceedings of the 6th Annual International Conference on Mobile Computing and Networking, pp 120-130, 2000.

[17] D. Son, A. Helmy, and B. Krishnamachari, "The Effect of Mobility-induced Location Errors on Geographic Routing in Ad Hoc Networks", IEEE Wireless Communications and Networking Conference (WCNC), 2004.

[18] Dongjin Son, Junghun Park, and Ahmed Helmy, "MobilityInduced Location Errors and its Effect on Geographic Routing in Ad Hoc Networks: Analysis and Improvement using Mobility Prediction", IEEE Transactions on Computing, Vol. ,3, No. 3, pp 233-245, 2004.
[19] B. Peng, R. Mautz, A. H. Kemp, W. Ochieng and Q. Zeng, "On the Effect of Localization Errors on Geographic Routing in Sensor Networks", IEEE International Conference on Communications, 3136-3140, 2008.

[20] B. Peng, R. Mautz, A. H. Kemp, W. Ochieng, and Q. Zeng, "On the Effect of Localization Errors on Geographic Routing in Sensor Networks", IEEE Transactions on Mobile Computing, Vol. 11, No. 9, 2011.

[21] Juzheng Li and Sol M. Shatz, "Toward Using Node Mobility to Enhance Greedy Forwarding in Geographic Routing for Mobile Ad Hoc Networks", In the International Workshop on Mobile Device and Urban Sensing, 2008.

[22] Xiaojing Xiang, Zehua Zhou, and Xin Wang, "Self-Adaptive On Demand Geographic Routing Protocols for Mobile Ad Hoc Networks", IEEE Transactions on Mobile Computing, Vol. 11, No. 9, pp. 1572-1586, 2012.

[23] Quanjun Chen, Salil S. Kanhere, and Mahbub Hassan, "Adaptive Position Update for Geographic Routing in Mobile Ad-hoc Networks", IEEE Transactions on Mobile Computing, Vol. 12, No.3, pp 489-501, 2013. 